\title{
TITLE:
}

\section{Über die Schrumpfung des Glasfadens beim Erhitzen}

$\operatorname{AUTHOR}(\mathrm{S})$ :

Sawai, Ikutaro; Ueda, Yoshihiro

CITATION:

Sawai, Ikutaro ...[et al]. Über die Schrumpfung des Glasfadens beim Erhitzen. 化學研究所學術報告 1929, 1

ISSUE DATE:

1929-11-30

URL:

http://hdl.handle.net/2433/74506

RIGHT: 


\title{
Über die Schrumpfung des Glasfadens beim Erhitzen.
}

\author{
Von Ikutaro SawaI und Yoshimiro Ueda. \\ Mit 12 Figuren im Text. \\ Einleitung.
}

Wie allgemein bekannt, verändert der Glasfaden seine Länge mit der Temperatur. Dieses Verhalten ergab handliche Mittel für die Bestimmung einiger Eigenschaften des Glases im hoch viskosen Zustand. BeRgGREN ${ }^{1}$ ) und später auch TAMmans u. a. ${ }^{2}$ ) benutzten die Erscheinung der Schrumpfung des Glasfadens bei hoher Temperatur als eine Methode für die Ermittlung der Werte der Oberflächenspannung des Glases, obwohl ihre Arbeiten gar keine systematischen betreffs der Schrumpfung waren. Weil aber dieses Phänomen im engen Zusammenhang mit der TammanN'schen Theorie ${ }^{3}$ ) der Schrumpfung der Glaslamellen steht, muB eine diesbezügliche Forschung von Interesse sein.

Nachdem wir über die Schrumpfung des Glasfadens durch die Spannung bei der Fadenherstellung berichtet hatten ${ }^{4}$ ), stellten wir noch einige Versuche über die Schrumpfung durch die Obertlächenspannung an, die kurz im folgenden mitgeteilt sind.

\section{Experimenteller Teil.}

1. Probe, Apparat und Methode.

Um Resultate von praktischer Bedeutung zu finden, haben wir als Versuchsmaterial das Sodakalkglas mit der Zusammensetzung von $75,00 \mathrm{SiO}_{2}, 2,83 \mathrm{Al}_{2} \mathrm{O}_{3}+\mathrm{Fe}_{2} \mathrm{O}_{3}, 6,43 \mathrm{CaO}$, und $15,40 \% \mathrm{Na}_{2} \mathrm{O}$ ausgewählt.

Die Fäden, die wir aus Glasstäben von obiger Zusammensetzung herstellten, wurden nach dem Durchmesser in 6 Klassen

1) B. Berganen, Ann. Phyg. (4) 44 (1914), 61.

2) G. TaMmanm und R. Tamke, Z. anorg. u. allg. Chem. 162 (1927), 1; G. Tammann und H. RaBe, Z. anorg. u, allg. Chem. 162 (1927), 17.

3) G. Tammann, Gött. Nachr. Math.-Phys. Klasse, 1912, 559.

4) I. Sawai und O. Morisawa, Z. anorg. u. allg. Chem. 173 (1928), 361. 
$(0,316,0,271,0,181,0,136,0,100$ und $0,031 \mathrm{~mm})$ geteilt und im verschlossenen Glasrohre aufbewahrt.

Fig. 1 gibt den Hauptteil des Versuchsapparats wieder. Ein Quarzrohr $Q$, an dessen unterem Teile der Haken $H$ befestigt ist, an dem der Faden hängt, ragt durch einen Korkstöpsel $C$ in ein von Hartglas verfertigtes Glasrohr $G$ hinein. Eine solche Einrichtung war zwecks der Verhinderung der Bewegung des Fadens durch einen etwaigen Luftstrom sehr nützlich. Der Reiter $w_{1}$, der durch den am unteren Eude des Fadens hangenden Haken $w_{2}$ gehängt ist, hat

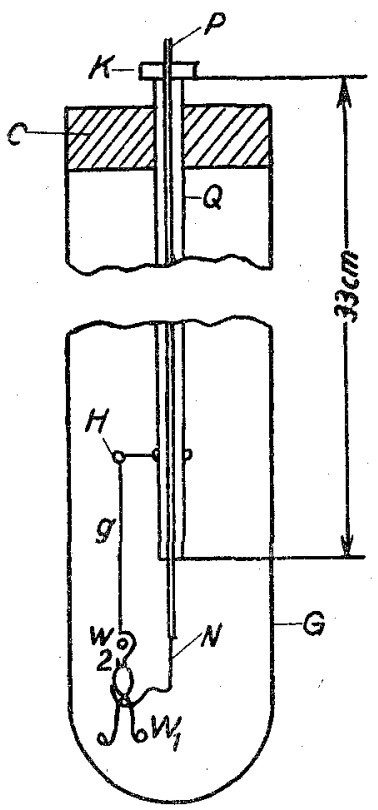

Fig. 1. den Zweck der Vermeidung der Schrumpfung des Fadens vor dem Beginn der Beobachtung. Er ist mit einem schmalen Porzellanrohr $P$ und einem Haken $N$, deren Einstellung mittels des Korkstöpsels $K$ ausgeführt werden kann, wegnehmbar.

Die Erhitzung des Fadens geschah in einem elektrischen Muffelofen mit zwei Fenstern. Mit einer Linse wurde ein reales Bild des durch monochromatisches Licht beleuchteten Fadens in dem Mikroskop abgebildet. Die Bewegung des Bildes wurde durch ein Okularmikrometer, bei dem ein Skalenteil $0,053 \mathrm{~mm}$ entsprach, gemessen. Für die Temperaturmessung wurde ein geeichtes Thermoelement von $\mathrm{Pt}$ und Pt-Rh benutzt.

Nachdem die Durchmesser eines Fadens von etwa $50 \mathrm{~mm}$ Länge an 3 Punkten gemessen waren, wurde er an den beiden Enden zur Perle geglüht, und danach seine Länge gemessen und abgewogen. Beim Versuche wurde der Faden mittels einer Perle an dem Haken $H$ (Fig. 1) eingehakt. An der anderen Perle wurde der abgewogene Haken $w_{2}$, der nach der Bedingung der Versuche 0,5-50 mg wog, eingehakt. Bei der Messung der Schrumpfungs- oder Verlängerungsgeschwindigkeit bei konstanter Temperatur wurde das Gewicht $w_{1}$ an dem Haken $w_{2}$ eingehakt. Das Gewicht $w_{1}$ wurde mittels des Hakens $N$ bis zum Beginn des Versuchs gestützt. Während des Versuchs wurde der Bewegungsabstand des Bildes aller 5 Minuten, eventuell aller 15 Sekunden notiert. 
2. Die Temperatur des Beginns der Schrumpfung.

Wie im Falle der Borbleigläser, beobachteten wir beim Sodakalkglas auch zwei Schrumpfungen $a$ und $b$, wie in Fig. 2 gezeigt ist.

Die bei der niedrigeren Temperatur stattfindende Schrumpfung $a$ verschwindet aber durch die Erhitzung bei $500^{\circ}$ für 30 Minuten. Also muB der Faden $\mathrm{Im}$ vorliegenden Falle durch einstündige Erhitzung bei $500^{\circ}$ behandelt werden. Darauf kommt nur eine Schrumpfung $b$ zum Vorschein. Der Kürze halber werden wir nun die Temperatur des Beginns der Schrumpfung $\left(t_{s}\right.$ in Fig. 2 als Schrumpfungspunkt bezeichnen. Falls die Erhitzungsgeschwindigkeit größer ist als $3^{0}$ pro Minute, so tritt nur ein Punkt $t_{s}$ auf, falls sie aber kleiner ist als $2^{\circ}$ pro Minute, so treten die zwei Punkte $t_{s_{1}}$ und $t_{s_{2}}$ mit einem Intervall ron $10-20^{\circ}$ für keine Längenänderung vor. Wenn bei unseren Versuchen keine Bemerkung angegeben ist, so stieg die Temperatur treppenartig an, und zwar so, dab die Temperatur erst

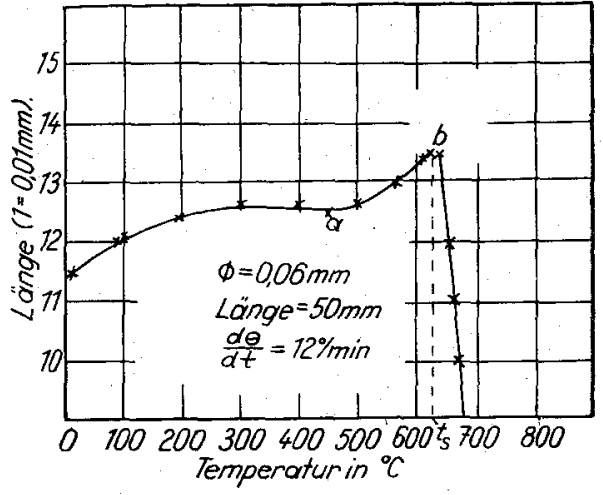

Fig. 2. für 10. Minuten mit der Geschwindigkeit von $1^{0}$ pro Minute anstieg, worauf sie für 10 Minuten lang konstant gehalten wurde, und dann erst wurde sie wieder zum Ansteigen gebracht. Dabei beobachteten wir stets zwei Schrumpfungspunkte: diese Punkte $\left(t_{s_{1}}\right.$ und $\left.t_{s_{2}}\right)$ liegen in einem verhältnismäBig engen Temperaturbereiche, wie man aus Tabelle 1 leicht ersehen kann. In Tabelle 1 sind die Lagen der Scbrumpfungspunkte in 3 Temperaturbereichen, die bei den unter verschiedenen Bedingungen ausgeführten 30 Versuchen beobachtet wurden, angegeben. In T'abelle 2 sind die 9 Beispiele aus den obigen Resultaten entnommen, und dadurch kann man die Einflüsse von Last und Durchmesser übersehen.

Tabelle 1.

\begin{tabular}{|c|c|c|c|c|}
\hline & & \multicolumn{3}{|c|}{ Temperatur in ${ }^{\circ} \mathrm{C}$} \\
\hline & & $530-580$ & $580-620$ & $600-620$ \\
\hline 茜 & $\begin{array}{l}t_{s_{1}} \\
t_{s_{z}}\end{array}$ & $\begin{array}{r}18 \\
3\end{array}$ & $\begin{array}{l}18 \\
15\end{array}$ & $\begin{array}{r}2 \\
12\end{array}$ \\
\hline
\end{tabular}


Tabelle 2.

\begin{tabular}{c|c|c|c|c}
\hline \hline Durchmesser in mm & $W^{1}$ ) in $\mathrm{mg}$ & $W / \pi r^{21}{ }^{11}$ & $t_{s_{1}}$ in ${ }^{0} \mathrm{C}$ & $t_{s_{2}}$ in ${ }^{0} \mathrm{C}$ \\
\hline \hline 0,316 & 7,2 & 92,0 & 571 & 587 \\
0,316 & 15,7 & 200,5 & 567 & 617 \\
0,316 & 16,1 & 205,5 & 587 & 612 \\
\hline 0,181 & 3,5 & 137,0 & 577 & 587 \\
0,181 & 4,3 & 168,0 & 572 & 587 \\
0,181 & 7,7 & 300,8 & 587 & 597 \\
\hline 0,100 & 0,5 & 64,0 & 552 & 555 \\
0,100 & 1,1 & 127,5 & 557 & 562 \\
0,100 & 5,9 & 752,5 & 597 & 607 \\
\hline
\end{tabular}

Wie aus dieser Tabelle ersichtlich, werden die Temperaturen der Schrumpfung mit der Last ebenso wie mit dem Durchmesser des Fadens höher.

Tabelle 3.

\begin{tabular}{c|c|c|c|c|c}
\hline \hline Durchmesser in mm & $O$ in mm & $M_{0}{ }^{3}$ in $\mathrm{mg}$ & $M_{0} / \pi r^{2}$ & $t_{s_{1}}$ & $t_{s_{2}}$ \\
\hline 0,271 & 30,00 & 4,8 & 8,4 & 582 & 597 \\
0,271 & 50,10 & 7,4 & 12,9 & 577 & 597 \\
0,271 & 70,00 & 9,0 & 15,7 & 577 & 587 \\
0,271 & 89,83 & 14,0 & 25,6 & 597 & 607 \\
\hline 0,136 & 30,26 & 1,3 & 9,0 & 577 & 587 \\
0,136 & 50,12 & 1,7 & 11,8 & 562 & 577 \\
0,136 & 70,43 & 2,4 & 16,8 & 562 & 582 \\
0,136 & 89,57 & 3,7 & 25,6 & 577 & 587 \\
0,100 & 30,10 & 0,4 & 5,1 & 547 & 572 \\
0,100 & 50,01 & 0,5 & 6,4 & 552 & 557 \\
0,100 & 7031 & 1,3 & 16,6 & 542 & 557 \\
0,100 & 90,58 & 1,5 & 19,2 & 552 & 557 \\
\hline
\end{tabular}

Um die Einflüsse der Länge und des Durchmessers vergleichen zu können, wurde der unbelastete Faden bei verschiedenen Längen erhitzt. Die Resultate werden in Tabelle 3 wiedergegeben. Wie die Tabelle klar zeigt, übt die Veränderung der Länge des Fadens auf den Schrumpfungspunkt einen geringen Einflub aus, obwohl dessen Gewicht pro Flächeneinheit, wie es in Tabelle 3 mit $Y_{0} / \pi r^{2}$ angezeigt ist, mit der Zunahme der Fadenlänge stetig größer wird. Der Durchmesser dementgegen übt einen stärkeren EinfluB auf den Schrumpfungspunkt aus. Der Schrumpfungspunkt $t_{s_{1}}$ des Fadens vom Durchmesser von $0,034 \mathrm{~mm}$ war $542^{\circ}$. Es ist interessant und

1) Mit $W$ wird das Gewicht des Fadens zusammen mit dem der Last bezeichnet.

2) $Y_{0}$ bedentet das Gewicht des Fadens allein. 
wichtig, daß die Fäden mit dem Durchmesser von 0,100 und $0,181 \mathrm{~mm}$ bei $542^{\circ}$ durch die Konstanthaltung der Temperatur die stetige Schrumpfung erweisen.

Tabelle 4.

\begin{tabular}{c|c|c|c|c}
\hline Durchmesser in mm & $d \theta / d t$ in ${ }^{0} \mathrm{C} /$ Min. & $t_{s_{1}}$ in ${ }^{\circ} \mathrm{C}$ & $t_{s_{2},}$ in ${ }^{0} \mathrm{C}$ & $M_{0}$ in $\mathrm{mg}$ \\
\hline 0,100 & 0,5 & 557 & 562 & 0,8 \\
0,100 & 1 & 557 & 582 & 1,0 \\
0,100 & 3 & 597 & 597 & 1,0 \\
0.100 & 10 & 617 & 617 & 1,2
\end{tabular}

Wie oben gesagt, übt die Erhitzungsgeschwindigkeit einen Einfluß auf den Schrumpfungspunkt aus. In Tabelle 4 werden einige Beispiele in dieser Beziehung wiedergegeben.

Ist die Last eines Fadens leichter als ein bestimmtes Gewicht, so schrumpft er, aber ist sie schwerer als dieses Gewicht, so verlängert er sich beim Erhitzen; z. B. die Fäden mit den Durchmessern von 0,316 bis zu $0,136 \mathrm{~mm}$ und von $50 \mathrm{~mm}$ Länge schrumpfen bei einer Last von $4 \mathrm{mg}$, aber sie verlängern sich bei einer Last von $5 \mathrm{mg}$. Ein Faden mit dem Durchmesser von $0,100 \mathrm{~mm}$ und $50 \mathrm{~mm}$ Länge schrumpft bei der Last von $3 \mathrm{mg}$, während er sich bei einer Last von $4 \mathrm{mg}$ verlängert.

Der mit der Last von 3 oder $4 \mathrm{mg}$ belastete Faden weist keine Verlängerung auf bei der Erhitzung bis etwa $800^{\circ}$, aber bei dieser Temperatur zerbricht er plötzlich. Wenn der Durchmesser so dick ist, daß das Gewicht des unbelasteten Fadens über $30 \mathrm{mg}$ beträgt, so liegen die Verhältnisse ganz anders. In diesem Falle gibt es 3 Arten der Längenänderung: 1. falls die Länge des Fadens kürzer als $30 \mathrm{~mm}$ (Durchmesser 0,7 mm, Gewicht 25,0 $\mathrm{mg}$ ) ist, so schrumpft er zu einer Perle zusammen; 2. falls

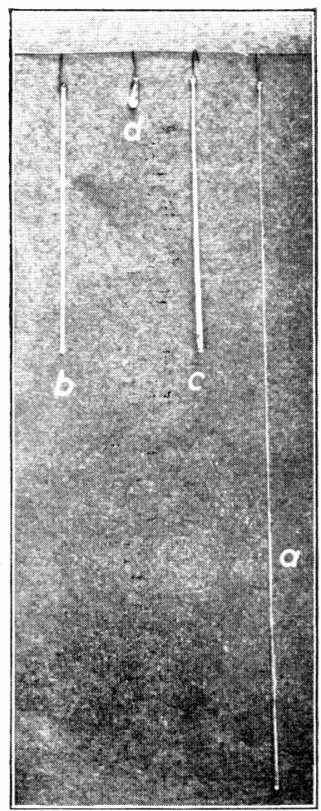

Fig. 3. $a$ Vor der Erhitzung, $b$ Zustand $1, c$ Zustand 2 , d Zustand 3. die Länge $40-60 \mathrm{~mm}(34,0$ bis $57,9 \mathrm{mg})$ ist, so schrumpft er bei $600-700^{\circ}$, und dann verlängert er sich bei $800^{\circ}$; 3. falls er länger als $70 \mathrm{~mm}(59,5 \mathrm{mg})$ ist, so verlängert er sich beim Erhitzen. In Fig. 3 findet man bei $b(1), c(2)$ und $d(3)$ die Gestalt der bei $800^{\circ}$ aus 
dem Ofen heraus genommenen Fäden, wo das untere Ende des Fadens bei $b$ etwas größer als bei $a$ ist.

Die Ausdehnungskoeffizienten der mit verschiedenen Lasten be. lasteten Fäden von verschiedenem Durchmesser wurden gemessen. Die Resultate der Messung ergaben fast konstante Werte, wie sie in Tabelle 5 wiedergegeben sind.

Tabelle 5 .

\begin{tabular}{|c|c|c|c|c|}
\hline $\begin{array}{l}\text { Durchmesser } \\
\text { in } \mathrm{mm}\end{array}$ & $\begin{array}{c}E \cdot g \\
\text { in }{ }^{\circ} \mathrm{C} / \mathrm{Min} .\end{array}$ & $W$ in $\mathrm{mg}$ & bei $\stackrel{r \cdot 10^{5}}{500-600^{0} \mathrm{C}}$ & Beinerk. \\
\hline 0,316 & 3 & 15,0 & 3,1 & Verlängert \\
\hline 0,180 & $\mathbf{3}$ & 11,9 & 4,1 & , \\
\hline 0,100 & 3 & 4,7 & 3,0 & \\
\hline 0,316 & 3 & 15,1 & 3,5 & Geschrumpft \\
\hline 0,181 & $\mathbf{3}$ & 9,0 & 4,6 & the \\
\hline 0,100 & 3 & 3,8 & 4,3 & 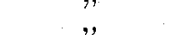 \\
\hline 0,316 & 3 & 12,4 & 3,9 & , \\
\hline 0,180 & 3 & 3,8 & 4,8 & " \\
\hline 0,100 & $\mathbf{3}$ & 0,8 & 3,8 & 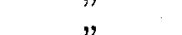 \\
\hline
\end{tabular}

3. Schrumpfungs- und Verlängerungsgeschwindigkeit.

Um die Schrumpfungs- und Verlängerungsgeschwindigkeiten bei einer konstanten Temperatur zu bestimmen, wurde der Faden 1 Stunde lang bei dieser Temperatur entspannt; danach wurde das Gewicht $w_{i}$ aus dem Gewicht $w_{2}$ (Fig. 1) weggenommen, darauf die Bewegung des Fadenbildes durch das Mikroskop gemessen.

Die Schrumpfungsgeschwindigkeit ist von der Temperatur, von dem Durchmesser und auch von der Last sehr abhängig. Diese Beziehungen werden unten in 4 Figuren mit 12 Beispielen graphisch dargestellt.

In Fig. 4a werden die Einflüsse der Temperatur dargestellt; in Fig. $4 \mathrm{~b}$ die des Durchmessers; in Fig. $4 \mathrm{c}^{1}$ ) die der Last auf die Schrumpfungsgeschwindigkeit, und endlich in Fig. $4 \mathrm{~d}$ die der Last und der Temperatur auf die Verlängerungsgeschwindigkeit. Man kann daraus ersehen, daB die Geschwindigkeit direkt mit der Temperatur und indirekt mit der Last und dem Durchmesser proportional ist.

Die Beziehung zwischen der Zeit und der Längenänderung ergaben die folgenden GesetzmäBigkeiten:

1) Die geklammerten Zahlen in Fig. 4 von $a$ zu $b$ ergeben die Werte von $\frac{M_{0}}{2}+m$. 
1. Bei mäBiger Geschwindigkeit besteht die Beziehung

$$
\frac{1}{t} \log \frac{l_{0}}{l}=K_{1},
$$

wo $t$ die Zeit, $l_{0}$ die Anfangslänge, $l$ die Länge nach der Zeit $t$, und $K$ eine Konstante ist.

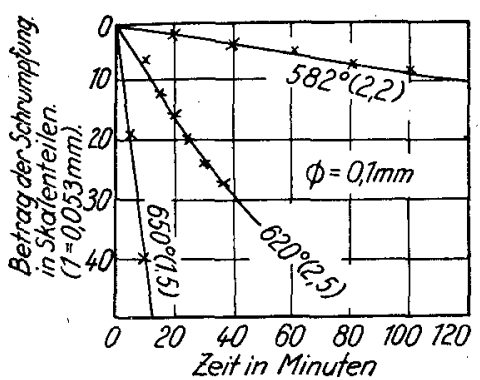

Fig. 4 a.

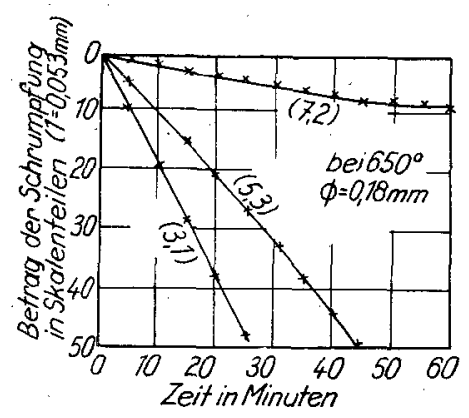

Fig. $4 \mathrm{e}$.

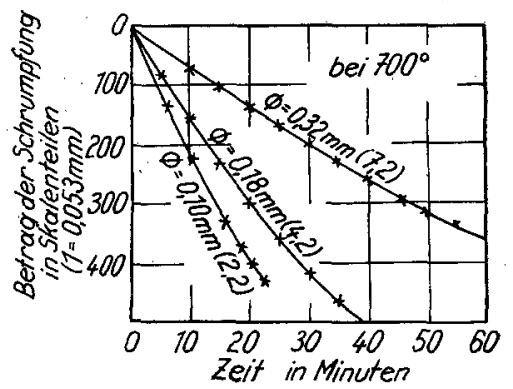

Fig. $4 \mathrm{~b}$.

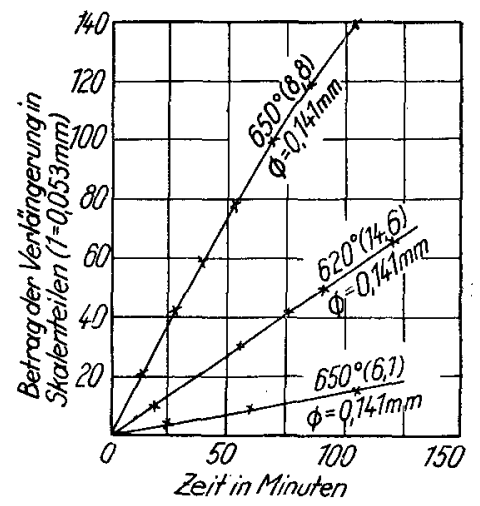

Fig. 4 d.

2. Wenn die Geschwindigkeit sehr klein ist, so wird die Geschwindigkeit der Längenänderung mit dem Verlauf der Zeit nach und nach kleiner, so dab die obige Beziehung durch die Formel

$$
\frac{1}{\sqrt{t}} \log \frac{l_{0}}{l}=K_{2}
$$

gut wiedergegeben werden kann. Einige Beweise dafür findet man in Tabelle 6. 
Tabelle 6.

\begin{tabular}{|c|c|c|c|c|c|c|}
\hline \multirow[b]{2}{*}{ Temp. in ${ }^{\circ} \mathrm{C}$} & \multicolumn{3}{|c|}{ Schrumpfung } & \multicolumn{3}{|c|}{ Verlängerung } \\
\hline & 620 & 650 & 700 & 620 & & \\
\hline Durchm. in mm & \multicolumn{3}{|c|}{0,181} & 0,141 & \multicolumn{2}{|c|}{0,141} \\
\hline$\frac{M_{0}}{2}+m$ in mg & 3,8 & 3,1 & 4,0 & 15,8 & \multicolumn{2}{|c|}{6,8} \\
\hline Zeit in Min. & $K_{1} \cdot 10^{4}$ & $K_{1} \cdot 10^{4}$ & $K_{1} \cdot 10^{3}$ & $K_{1} \cdot 10^{4}$ & $K_{1} \cdot 10^{3}$ & $K_{2} \cdot 10^{3}$ \\
\hline$\tilde{s}$ & 1,8 & 8,6 & 8,0 & & & \\
\hline 10 & 1,6 & 9,0 & 7,7 & 2,3 & 1,1 & 3,6 \\
\hline 15 & $1, \mathfrak{3}$ & 8,9 & 7,8 & & & \\
\hline 20 & 1,8 & 9,3 & 7,9 & 2,3 & 0,9 & 4,0 \\
\hline 25 & 1,5 & 9,8 & 8,0 & & & \\
\hline 30 & 1,5 & 8,9 & 8,2 & 2,4 & 0,8 & 4,3 \\
\hline 85 & 1,5 & 8,9 & 8,1 & & & \\
\hline 40 & 1,5 & 9,1 & 8,0 & 2,4 & 0,7 & 4,3 \\
\hline 45 & 1,5 & 7,7 & & & & \\
\hline 50 & 1,5 & 8,9 & & 2,4 & 0,6 & 4,3 \\
\hline 55 & 1,5 & 8,9 & & & & \\
\hline 60 & 1,5 & 8,8 & & 2,4 & 0,5 & 4,2 \\
\hline
\end{tabular}

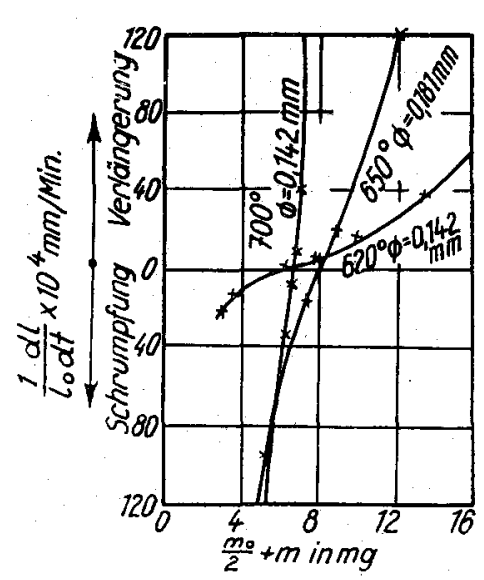

Fig. 5 .

Die Beziehung zwischen der $\frac{M_{0}}{2}+m$ und der Geschwindigkeit bei $600--700^{\circ}$ für die Fäden von $50 \mathrm{~mm}$ Länge sind in Fig. 5 graphisch dargestellt. Wie die Kurven klar zeigen, nehmen die Kurven eine S-förmige Gestalt an mit zwei Knicken, und zwar vergrößert sich der Abstand zwischen beiden Knicken mit aufsteigender T'emperatur. Diese Knicke, welche man auch aus ähnlichen Kurven von Trouton ${ }^{1}$ ) und BerGGReN ${ }^{2}$ ) ersehen kann, ergeben das Gewicht der

Last. Die Beziehung zwischen der Zeit und der Längenänderung kann mit der Formel

$$
\frac{1}{t} \log \frac{l_{0}}{l}=K_{l}
$$

gut ausgedrückt werden. Wir haben beobachtet, daB die obige Beziehung beim Faden mit dem Durchmesser von $0,147 \mathrm{~mm}$ und $50 \mathrm{~mm}$

1) F. T. Trouton, Proc. Roy. Soc. 77 (1906), 426.

2) B. Berggren, l. c. 
Länge erst mit der Last von $25,3 \mathrm{mg}$ bei $582^{\circ}$, und mit $200 \mathrm{mg}$ bei $550^{\circ}$ erfüllt ist.

Über $700^{\circ}$ wurde die Bewegung des Fadens sehr schnell, so daB, um ihn zur Glasperle schrumpfen zu lassen, Erhitzung bei $750^{\circ}$ für 25 Minuten, bei $800^{\circ}$ für 6 Minuten und bei $850^{\circ}$ für 3 Minuten genug war.

\section{Berechnung der Werte der Oberflächenspannung und der Zähigkeit.}

Aus dem Resultat der Messung der Schrumpfungs- und Verlängerungsgeschwindigkeiten kann man nach folgender Weise die Werte der Oberflächenspannung und Zähigkeit berechnen.

Es sei, wie in Fig. 6, ein Faden vom Gewicht $Y_{0}$, Länge $l$, und Radius $r$ mit der Last $m$ behangen. Die Kraft $p$, welche an der Flächeneinheit im Abstand $x$ vom oberen Ende angreift, wird mit der Formel

$$
p=\frac{1}{\pi r^{2}}\left[(2 \pi r c-m)-(l-x) \rho g \pi r^{2}\right]
$$

ausgedrückt. Hier bezeichnet $a$ die Oberflächenspannung, $o$ das spezifische Gewicht des Glases und $g$ die Erdschwerkraft. Nach Trouton $^{1}$ ) hat man bei der Bewegung zäher Körper die Gleichung

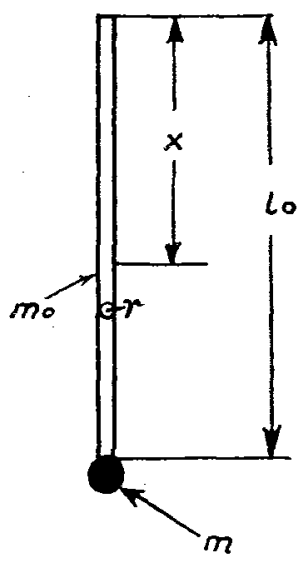

Fig. 6.

$$
\frac{d v}{d x}=\frac{1}{i} p
$$

als die mathematische Beziehung zwischen der Geschwindigkeit $v$ an dem Punkte $x$ und der über diese Flächeneinheit wirkenden Kraft $p$, wo $\lambda$ den sogenannten ,coefficient of viscous traction"s bedeutet. Also ist die Geschwindigkeit des unteren Endes des Fadens $\iota_{1}$ für den Faden ron der Länge $l$ gleich

$$
v_{l}=\frac{1}{\pi r^{2} \dot{\lambda}} \int_{0}^{l}\left[(2 \pi r \alpha-m)-(l-x) g \rho \pi r^{2}\right] d x,
$$

und auch die Beziehung gleich

$$
m m_{0}=g g \pi r^{2} l \text {. }
$$

1) F. T. Trocton, l. e. 
Bei der Längenänderung wird der Durchmesser des Fadens immer variieren. Um die Berechnung zu vereinfachen, kann man statt dieses wirklichen Durchmessers den mittleren Durchmesser $r_{m}$ mit dem Fehler kleiner als $1 \%$, benutzen. Der mittlere Durchmesser läbt sich leicht aus der Beziehung

$$
r_{m}=\frac{r_{0}\left(1+\sqrt{\frac{l_{0}}{l_{n}}}\right)}{2}
$$

berechnen, weil bei konstanter Temperatur

$$
\pi r^{2} l=\text { const. }
$$

ist. In der oberen Gleichung (5) bezeichnet $l_{0}$ die Länge des Fadens. beim Anfang des Versuchs, und $l_{e}$ dieselbe beim Ende und $r_{0}$ den Durchmesser beim Anfang des Versuchs. Aus (3), (4) und (כ) ergibt sich:

$$
\left.v_{l}=\frac{l}{\pi r_{m}^{2} \lambda} \Gamma_{L}\left(2 \pi r_{m} \alpha-m\right)-\frac{H_{0}}{2}\right] .
$$

Also bekommt man

oder

$$
-\frac{d l}{d t}=\frac{l}{\pi)_{m}^{2} \lambda}\left[2 \pi r_{m} \alpha-\left(m+\frac{M_{0}}{2}\right)\right]
$$

$$
-\ln l=\frac{t}{\pi r_{m}^{2} \lambda}\left[2 \pi r_{m} \alpha-\left(m+\frac{M_{0}}{2}\right)\right]+c .
$$

Mit der Bedingung

erhält man die Beziehung

$$
\left.\begin{array}{l}
l=l_{0} \\
t=0
\end{array}\right\}
$$

$$
2,303 \log \frac{l_{0}}{l}=\frac{t}{\pi r_{m}^{2} 2}\left[2 \pi r_{m} \alpha-\left(m+\frac{M_{0}}{2}\right)\right] .
$$

In der Gleichung (8) sind $l_{0}$ und $r_{0}$ die GröBen, die man unter dem Mikroskop messen kann, und $m, M_{0} / 2$ sind die GröBen, die man nach dem Versuche abwägen oder berechnen kann. Also gibt es 3 Methoden für die Berechnung der Oberflächenspannung, wie folgt:

1. Eliminieren von $\lambda$. - Unter anderen glauben wir die folgenden zwei Prozesse als die besten erkannt zu haben: a) durch die Messungen von verschiedenem $r_{n}$ und konstantem $m$ (Methode 1a); b) durch die Messungen bei verschiedenem $m$ und konstantem $r_{m}$ (Methode 1b)! 
2. Mittels ener Kurve, die die Beziehung zwischen $v_{1}$ und $\frac{M_{0}}{2}+m$ bezeichnet. - Aus dieser Kurve sucht man den zur Gleichung $v_{l}=0$ gehörenden Wert von $\frac{M_{0}}{2}+m$ auf, dessen Division durch $2 \pi r$ dem gesuchten Wert entspricht.

3. Ermittlung des Wertes $v_{x}=0$, wo keine Änderung des Durchmessers stattfindet. - Durch die Erhitzung wird der Durchmesser unterhalb des Fadens stärker wie in Fig. 3c, und der ober halb des Fadens schmaler als der ursprüngliche. Also kann man mittels des Komparators und Okularmikrometers die Durchmesser der verschiedenen Teile des Fadens messen. Aus diesen Messungen wird eine Kurve, die die Änderung des Durchmessers bezeichnet, konstruiert. Mittels dieser Kurve wird der Punkt, der nach der Erhitzung keine Änderung des Durchmessers aufweist $\left(v_{x}=0\right)$, aufgesucht. Ist das Gewicht des Fadens unterbalb des obengenannten Punkts mit $2 \pi r$ dividiert, so erhält man den Wert der Oberflächenspannung (Methode 3).

Tabelle 7.

\begin{tabular}{c|c|c|c|c}
\hline \multirow{2}{*}{$\begin{array}{c}\text { Temperatur } \\
\text { in }{ }^{\circ} \mathrm{C}\end{array}$} & \multicolumn{4}{|c}{$\alpha$ in $\mathrm{mg} / \mathrm{mm}$} \\
\hline & Methode 1 a & Methode 1 b & Methode 2 & Methode 3 \\
\hline 600 & 23,59 & 15,23 & 15,40 & - \\
620 & 14,30 & 14,51 & 14,06 & - \\
650 & 20,50 & 13,68 & 13,32 & - \\
700 & 15,56 & 14,00 & 15,06 & 13,25 \\
750 & - & 13,16 & 12,85 & 13,35 \\
800 & - & 11,34 & 11,35 & 11,81
\end{tabular}

Die Resultate der Berechnung werden in Tabelle 7 wiedergegeben. Obwohl die Methode 1a, wie man sieht, etwas abweichende Werte von $\alpha$ gibt, genügt sie doch noch, weil die Werte von $\lambda$ für die so abweichenden Werte der Geschwindigkeiten natürlich etwaige Abweichungen zeigen müssen. Die Methode 3 ist ebensogut bei höherer Temperatur wie bei niedrigerer Temperatur leicht

Tabelle 8.

\begin{tabular}{c|c|c|c}
\hline Temp. in ${ }^{0} \mathbf{C}$ & $\log \log \lambda$ & $\log \lambda$ & $\lambda$ \\
\hline 550 & 0,87 & 7,2 & $1,8 \cdot 10^{7}$ \\
580 & 0,83 & 6,8 & $5,8 \cdot 10^{6}$ \\
600 & 0,79 & 6,2 & $1,5 \cdot 10^{6}$ \\
620 & 0,73 & 5,4 & $2,3 \cdot 10^{5}$ \\
650 & 0,69 & 4,9 & $7,9 \cdot 10^{4}$ \\
700 & 0,57 & 3,7 & $5,2 \cdot 10^{3}$ \\
750 & 0,53 & 3,4 & $2,3 \cdot 10^{3}$ \\
800 & 0,46 & 2,9 & $7,4 \cdot 10^{2}$ \\
& & &
\end{tabular}


ausführbar, aber sie gibt über $800^{\circ}$ etwas abweichende Werte der Oberflächenspannung, weil die Zeitdauer des sich im Ofen Befindens zu kurz ist. Die Resultate der Berechnung von $\lambda, \log \lambda$ und $\log \log \lambda$ werden in Tabelle 8 verzeichnet.

Die Beziehungen zwischen $\log \lambda$ oder $\log \log \lambda$ und der Temperatur werden in Fig. 7 graphisch dargestellt.

Die Kurve von $\log \log \lambda$ ist aus zwei Linien zusammengesetzt, deren Werte unter $700^{\circ}$ durch die Formel

$$
\log \log \lambda_{t}=0,87-0,002(t-550)
$$

gut wiedergegeben werden können. Nach TRouToN ${ }^{1}$ ) wird die Beziehung zwischen $\lambda$ und dem Zähigkeitskoeffzienten $\mu$ durch $\mu=\frac{1}{3} \lambda$ bezeichnet. Also können wir annehmen, daB die obige lineare Beziehung den Grund für das Le Chatelien'sche Gesetz der Zähigkeit darstellt.

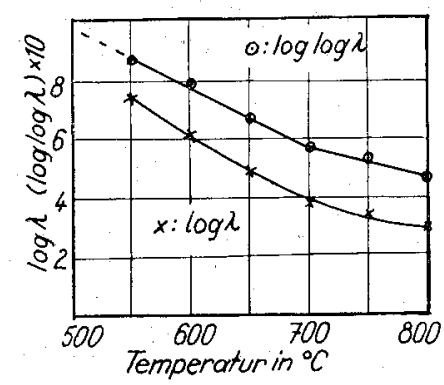

Fig. 7.

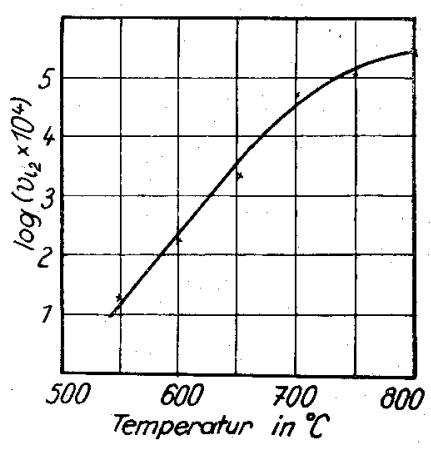

Fig. 8.

5. Bedingung der Schrumpfung.

Für die Fäden von $M_{0} / 20,4 \mathrm{mg}, \Phi=0,1 \mathrm{~mm}$ und $l=50 \mathrm{~mm}$ haben wir mittels der Gleichung (6) die Schrumpfungsgeschwindigkeit bei verschiedenen Temperaturen berechnet. Diese Resultate sind in Tabelle 9 zusammengestellt.

Tabelle 9.

\begin{tabular}{|c|c|c|c|c|c|c|}
\hline Temp. in ${ }^{\circ} \mathbf{C}$ & 550 & 600 & 650 & 700 & 750 & 800 \\
\hline $\begin{array}{c}V_{l} \text { in } \operatorname{mm} / \mathrm{Min} \\
\log V_{l} \cdot 10^{4}\end{array}$ & $\begin{array}{c}1,96 \cdot 10^{-3} \\
1,29\end{array}$ & $\begin{array}{c}1,90 \cdot 10^{-2} \\
2,28\end{array}$ & $\begin{array}{c}2,9 \cdot 10^{-1} \\
3,46\end{array}$ & $\begin{array}{l}5.1 \\
4,71\end{array}$ & $\begin{array}{c}10,3 \\
5,01\end{array}$ & $\begin{array}{r}27,2 \\
5,43\end{array}$ \\
\hline
\end{tabular}

Die Ergebnisse dieser Berechnung ergeben die annähernd richtigen Werte von $V_{l}$; z. B. bei $557^{\circ}$ war das Ergebnis der Berechnung durch Interpolieren $1,26 \cdot 10^{-3} \mathrm{~mm} / \mathrm{Min}$., während das Resultat der Messung $1,9 \cdot 10^{-3} \mathrm{~mm} /$ Min. ist. Die Beziehung zwischen der Temperatur und $\log V_{l}$ wird in Fig. 8 verzeichnet.

1) F. T. Trouton, l. e. 
Da kann man verstehen, daB die Werte von $V_{l}$ zwischen 500 bis $600^{\circ}$ rasch zunehmen. Dementgegen sind die Werte der Ausdehnungskoeffizienten $r$ fast dieselben, so daß sie bei $500-600^{\circ}$ etwa $3,6 \cdot 10^{-5}$ betragen. Also kann man den Betrag der thermischen Ausdehnung des Fadens infolge des Temperaturanstiegs für eine Zeiteinheit durch die Gleichung

$$
V_{l_{1}}=\operatorname{lr} \beta
$$

berechnen, wo $l$ die Länge des Fadens, $r$ den thermischen Ausdehnungskoeffizient, $\beta$ die Geschwindigkeit des Temperaturanstiegs bedeutet. Die Geschwindigkeit der Schrumpfung kann man mittels der Gleichung (6) wie

$$
V_{l_{2}}=\frac{1}{\pi r_{m}^{2} \lambda}\left[\left(2 \pi r_{m} \alpha-m\right)-\frac{M_{0}}{2}\right]
$$

berechnen. Die berechneten Resultate von $V_{l_{1}}$ und $V_{l_{2}}$ sind für einige Fälle in Tabelle 10 wiedergegeben. Vergleicht man diese beiden Werte, so kann man verstehen, daB $V_{l_{2}} \geqq V_{l_{1}}$ ist. Aber die Werte $V_{l_{2}}$ nehmen mit absteigender Temperatur so rasch ab, daB direkt unterhalb des Schrumpfungspunktes $V_{l_{1}}>V_{l_{2}}$ ist. Die Richtigkeit dieser Auffassung wird durch die fast konstanten Werte von $r$ bei verschiedenen Bedingungen bewiesen, und zwar haben wir bestätigt, daß die Werte von $V_{l_{2}}$ bei $540^{0}$ die Ordnung von $10^{-7} \mathrm{~mm} / \mathrm{Min}$. haben. Also der Tabelle 10 kann man leicht entnehmen, dab für die Schrumpfung die Bedingung $V_{l_{2}}>V_{l_{1}}$, und bei dem Schrumpfungspunkt die Beziehung $V_{l_{2}} \geqq V_{l_{1}}$, erfüllt sein muB.

Tabelle 10.

\begin{tabular}{c|c|c|c|c}
\hline$d \Theta / d \operatorname{tin}^{0} \mathrm{C} / \mathrm{Min} \cdot$ & $t s$ in ${ }^{0} \mathrm{C}$ & $V_{l_{1}}$ in $\mathrm{mm} /$ Min. & $V_{l_{2}}$ in mm/Min. & Verbältnisse \\
\hline \hline 0,5 & $557-562$ & $9,81 \cdot 10^{-4}$ & $1,19 \cdot 10^{-3}$ & 1,3 \\
1 & $557-582$ & $1,81 \cdot 10^{-3}$ & $2,47 \cdot 10^{-3}$ & 1,4 \\
3 & 595 & $5,40 \cdot 10^{-3}$ & $1,50 \cdot 10^{-2}$ & 2,8 \\
10 & 617 & $1,77 \cdot 10^{-2}$ & $4,01 \cdot 10^{-2}$ & 2,3
\end{tabular}

$\mathrm{Ob}$ der Faden schrumpft oder nicht, hängt von der Bedingung $\frac{M_{0}}{2}+m \geqq 2 \pi r \alpha$ und nicht $M_{0}+m \geqq 2 \pi r \alpha$ ab. Der Beweis da. für wird der Tabelle 11 entnommen. In Tabelle 11 sind die Werte von $W\left(=M_{0}+m\right)$ und $\frac{M_{0}}{\mathbf{2}}+m$ gegenübergestellt. Dabei wurde der Faden mit der Last von $4 \mathrm{mg}$ und $5 \mathrm{mg}$ behangen (bei dem Faden vom Durchmesser von $0,100 \mathrm{~mm}$ sind die Gewichte $3 \mathrm{mg}$ und $4 \mathrm{mg}$ ) 
Tabelle 11.

\begin{tabular}{c|c|c|c|c|c}
\hline$\Phi$ in mm & $W$ in mg & $\frac{M_{0}}{2}+m$ & $t_{8}$ in ${ }^{0} \mathrm{C}$ & $\frac{W}{2 \pi r}$ in $\mathrm{mg} / \mathrm{mm}$ & $\frac{1}{2 \pi r}\left(\frac{M_{0}}{2}+m\right)$ \\
\hline \hline \multirow{2}{*}{0,181} & 9,0 & 6,6 & 627 & 15,8 & 11,6 \\
\hline \multirow{2}{*}{0,136} & 6,8 & 5,7 & 657 & 15,9 & 13,5 \\
\hline \multirow{2}{*}{0,100} & 3,0 & 7,7 & Verlängert & 17,1 & 13,4 \\
\hline & 3,8 & 3,5 & 612 & 12,1 & 14,5 \\
\hline & 4,7 & 4,4 & Verlängert & 15,0 & 11,2 \\
\hline
\end{tabular}

Verglexcht man die letzten zwei Spalten in Tabelle 11, so kann man entnehmen, daB die Werte von $\frac{M_{0}}{2}+m$ zu beiden Seiten eines bestimmten Wertes (der der annähernde Wert von $\alpha$ ist) liegen. Die Werte von $W / 2 \pi r$ aber haben nicht dieselbe Beziehung. Wenn der Faden schrumpft, so muB $\alpha>\frac{1}{2 \pi r}\left(\frac{M_{0}}{2}+m\right)$ sein, und wenn er sich verlängert, so muß $\alpha<\frac{1}{2 \pi r}\left(\frac{M_{0}}{2}+m\right)$ sein. Aber die aus den Werten von Tabelle 11 angenommenen Werte von $\alpha$ sind stets kleiner als dieselben in Tabelle 7. Diese Tatsache kann dadurch erklärt werden, daß bel dem Schrumpfungspunkt $V_{l_{2}} \geqq V_{l_{1}}$ ist. Daher muB der Faden erst mit dem Gewicht $\frac{M_{0}}{2}+m$ schrumpfen, das ziemlich leichter ist als das mit (Tabelle 7) berechnete. Bei dem Faden von verhältnismäßig gröBerem Durchmesser gab es etwas kleinere Werte von $\frac{1}{2 \pi r}\left(\frac{M_{0}}{2}+m\right)$ als beim schmalen Faden. Diese Erscheinung kann man auch dadurch erklären, daß der Durchmesser. des oberen Teils des Fadens schmaler werden kann, bevor er mit mäBiger Geschwindigkeit zu schrumpfen beginnt. Also betreffs der Bedingung der Schrumpfung kann man angeben:

1. $2 \pi r \alpha\left(\frac{M_{0}}{2}+m\right)$;

2. der Durchmesser des Fadens darf nicht zu schmal werden bevor dessen Schrumpfungsgeschwindigkeit größer als $V_{l_{1}}$ wird.

\section{Weitere Versuche.}

Bei $600^{\circ}$ beobachteten wir das interessante Phänomen, daB nur bei dieser Temperatur der einmal verlängerte Glasfaden umgekehrt 
mit dem Verlauf der Zeit zu schrumpfen beginnt. In Fig. 9 ist diese Erscheinung an zwei Beispielen graphisch dargestellt. Diese Schrumpfung ist etwas anders als die bisher von uns beobachteten. Bei dieser Schrumpfung ist die Verminderung der Länge auch von der Verkleinerung des Durchmessers begleitet. Der Faden, der beim Beginn der Versuche einen Durchmesser von 0,14 mm hatte, verminderte ihn am Ende bis zu $0,12 \mathrm{~mm}$, und das Resultat der Berechnung aus der Verminderung seiner Länge war $0,13 \mathrm{~mm}$. Ursache dieser Schrumpfung ist nicht irgendeine Veränderung im physikalischen Zustand, weil die Beobachtung unter einem Ultramikroskop mit Paraboloidkondensor keine Änderung seines Zustandes erwies, und auch das Resultat der LaUE'schen Röntgenaufnahme ${ }^{1}$ ) einen Beweis, daB der Faden ganz amorph ist, ergab. Also muB vermutet werden, daB die Ursache dieser

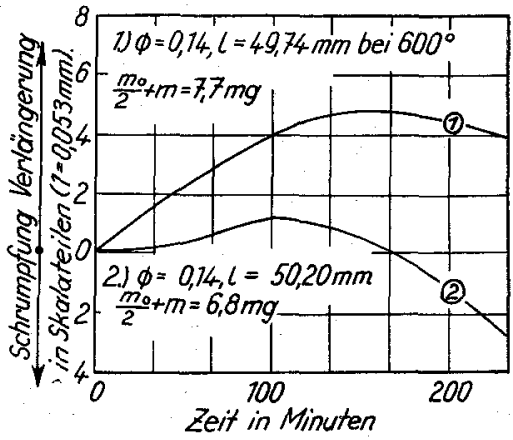

Fig. 9. Volumenverminderung in einer Änderung des Moleküls, aus dem das Glas gebildet ist, liegt.

\section{Theoretischer Teil.}

Betreffs des Schrumpfungspunkts der Glaslamelle hat TAMMANN ${ }^{2}$ ) eine Theorie mitgeteilt, die aus dem Zusammenhang zwischen der Oberflächenspannung und der Festigkeit für eine Lamelle einen bestimmten Schrumpfungspunkt gibt. BERGGREN ${ }^{3}$ ) betrachtete diese Schrumpfung als ein FlieBen, dessen Geschwindigkeit durch die Oberflächenspannung (samt etwaigen äußeren Kräften), teils durch die Zähigkeit des Glases bestimmt wird. Es existiert nach dieser Auffassung kein bestimmter Schrumpfungspunkt.

Bei unserem Falle liegt der Schrumpfungspunkt etwas über dem Entspannungspunkt des Glases. Also ist es klar, daB sich die Spannung, die in dem Faden stattfindet, mit dem Verlauf der Zeit durch die relative Bewegung innerhalb des Fadens verschwindet. Erhitzt man den Glasfaden, so verlängert er sich durch die ther-

1) 50 K.V. 70 stündige Beleuchtung.

2) G. Tamitann, 1. e.

3) B. Bergaren, I. c. 
mische Ausdehnung, obwohl durch die Oberflächenspannung längsgerichteter Druck ausgeübt wird. Also existiert während der Erhitzung eine längsgerichtete Spannung im Faden.

Ist die Temperatur des Fadens zu niedrig und die Zähigkeit zu groB, daB die Geschwindigkeit der Schrumpfung kleiner als die Geschwindigkeit der thermischen Ausdehnung ist, so beobachtet man während der Erhitzung nur die Verlängerung. Bei dieser Temperatur aber muß der Faden schrumpfen, wenn die Temperatur konstant erhalten wird. Die Bestätigung dieses Schlusses wurde schon anfangs beschrieben. Also ist es klar, daB der Schrumpfungspunkt, wie üblich, bei der Erhitzung des Fadens beobachtet wird, nicht der Punkt, bei dem die Kurve der Oberflächenspannung und die der Zähigkeit sich schneiden. Außerdem haben wir bestätigt, da $\beta$ bei dem Schrumpfangspunkt zwei Bedingungen, d. h. $V_{l_{2}} \geqq V_{l_{1}}$, $2 \pi r \kappa>\left(\frac{M_{0}}{\underline{\underline{ }}}+m\right)$ erfüllt werden müssen, und diese Bedingungen hängen nur von der Schrumpfungsgeschwindigkeit des Fadens ab. Also glauben wir, daß es richtig ist, die mit dem Schrumpfungspunkt zusammenhängenden Erscheinungen als ein Geschwindigkeitsproblem zu betrachten. Die Tatsache, daB die Schrumpfungspunkte in einem engen Temperaturbereiche liegen, kann man durch die mit der Temperatur rasch zunehmende Geschwindigkeit $V_{l_{2}}$ und ihre Abhängigkeit von den äußeren Bedingungen leicht erklären.

Erhitzt man den Glasfaden, um seine Längenänderung $\mathrm{zu}$ beobachten, so wird man die folgenden 3 Fälle finden:

1. Der Faden schrumpft zur Glasperle zusammen.

2. Er verlängert sich nach der einmaligen Schrumpfung.

3. Er verlängert sich nur während der Erhitzung.

Die Bedingungen dieser 3 Fälle kann man formulieren wie folgt: Die Spannung $p_{0}$, die am obersten Teil des Fadens ausgeübt wird, ist

$$
p_{0}=\frac{2 \alpha}{r}-\frac{1}{\pi r^{2}}\left(M_{0}+m\right),
$$

worin $M_{0}$ das Gewicht des Fadens, und $m$ das der Last ist. Also:

1. Sei die Beziehung $\frac{2 \alpha}{r}>\frac{1}{\pi r^{2}}\left(M_{0}+m\right)$ bis zum mobilen $\mathrm{Zu}$ stand erfüllt, so wird der Durchmesser während der Erhitzung immer vergrößert, bis der Faden zur Perle umgeformt wird.

2. Für die Erscheinung 2 kann man folgende zwei Bedingungen aufstellen; nämlich a) sei der Temperaturkoeffizient der Oberflächen- 
spannung so groB, daB bei niedriger Temperatur $\frac{2 \alpha}{r}>\frac{1}{\pi r^{2}}\left(M_{0}+m\right)$, aber bei höherer Temperatur $\frac{2 \alpha}{r}<\frac{1}{\pi r^{2}}\left(M_{0}+m\right)$ ist, so kann man den Wert derselben durch die Schrumpfungsmethode ermitteln; b) am oberen Teil des Fadens $\frac{2 \alpha}{r}<\frac{1}{\pi r^{2}}\left(M_{0}+m\right)$, dab ihr Durchmesser sich verringert. Bei dem von uns genommenen Glas ist der Temperaturkoeffizient so klein, daB es uns die Beziehung b) vermuten läBt. Bei diesem Fall kann man die Beziehung nicht mit einer einfachen mathematischen Formel ausdrücken.

3. Für die Erscheinung 3 könnte es auch zwei Bedingungen geben: a) ist die Last so schwer, daB an dem unteren Ende $\frac{2 \alpha}{r}<\frac{M}{\pi r^{2}}$ ist, so wird man nur die Verlängerung während der Erhitzung beobachten. b) ist der Durchmesser so groß und das Gewicht so schwer, daß die Verlängerungsgeschwindigkeit an der Oberhälfte des Fadens immer größer als die Schrumpfungsgeschwindigkeit an der Unterhälfte desselben ist, so beobachtet man im ganzen nur die Verlängerung.

\section{Zusammenfassung.}

1. Systematische Versuche über die Längenänderung des Glasfadens beim Erhitzen wurden dargestellt.

2. Die Temperatur des Beginns der Schrumpfung ist von dem Durchmesser des Fadens, von der an ihm hängenden Last, und auch von der Erhitzungsgeschwindigkeit sehr abbängig.

3. Eine Methode für die Messung der Oberflächenspannung wurde dargestellt und die Resultate der Messungen wurden mitgeteilt.

4. Einige theoretische Betrachtungen über den Schrumpfungspunkt sowie über die Bedngung der Umformung des Fadens wurden angestellt.

Es ist uns eine angenehme Pflicht, Herrn Prof. Dr. M. CHIKASHIGE für seine lebhaften Anregungen zu obigen Untersuchungen unseren herzlichsten Dank auszusprechen.

Kioto (Japan), Universität, Institut für chemische Untersuchung.

Bei der Redaktion eingegangen am 28. Januar 1929. 